\title{
A Case Report on the Effect of Everolimus in Renal Angiomyolipoma Associated With Tuberous Sclerosis Complex
}

\author{
Huma M Paika ${ }^{\mathrm{a}, \mathrm{d}}$, Yull E. Arriaga ${ }^{\mathrm{b}}$, Anthony Setiawan ${ }^{\mathrm{c}}$
}

\begin{abstract}
Tuberous Sclerosis (TS) is a well known disorder which manifests with benign tumors in various organ systems, including angiomyolipomas (AML) of the kidneys. The mTOR inhibitors have emerged as a potential systemic treatment strategy to halt the growth of renal angiomyolipomas and to prevent life threatening complications such as bleeding. This report shows that everolimus treatment may have an impact on size and density of renal AML lesions which may lead to prevention or delay the development of renal cell carcinoma as well as prevent bleeding from the renal AML lesions. A 20-year-old Hispanic female and a 24-year-old Asian female both with sporadic TS were administered daily oral everolimus therapy. Both showed stability in their renal AML lesions and notable improvement of their extra-renal TS manifestations. Treatment with daily everolimus may lead to reduction in size and/or decreases in density or renal AML lesions. This may have an impact on the risk of developing renal cell carcinoma as well as preventing bleeding from renal AML lesions.
\end{abstract}

Keywords: mTOR inhibitor; Tuberous sclerosis; Angiomyolipoma; Everolimus

\section{Introduction}

Tuberous Sclerosis (TS) is a well known disorder which

Manuscript accepted for publication January 14, 2014

${ }^{a}$ Department of Internal Medicine, University of Texas Southwestern Medical Center, 5323 Harry Hines Boulevard, Dallas, Texas, 75390-8852, USA

${ }^{\mathrm{b}}$ Division of Hematology Oncology, University of Texas Southwestern Medical Center, 5323 Harry Hines Boulevard, Dallas, Texas, 75390-8852, USA

${ }^{c}$ Department of Radiology, University of Texas Southwestern Medical Center, 5323 Harry Hines Boulevard, Dallas, Texas, 75390-8852, USA

${ }^{\mathrm{d}}$ Corresponding author: Huma M Paika, Department of Internal

Medicine, University of Texas Southwestern Medical Center, 5323

Harry Hines Boulevard, Dallas, Texas, 75390-8852, USA.

Email: Huma.paika@phhs.org

doi: http://dx.doi.org/10.14740/jmc1665w manifests with benign tumors in various organ systems, including tubers and subependymal giant cell astrocytomas (SEGA) of the brain, lymphangiomyomatosis (LAM) of the lungs, rhabdomyomas of the heart, facial angiofibromas, as well as angiomyolipomas (AML) of the kidneys [1]. It afflicts nearly 40,000 individuals in the USA [2]. Nearly $80 \%$ of patients with TS have renal manifestations [3]. TSC1 and TSC2 are tumor suppressor genes. In patients with TS, a germ line mutation is usually present in TSC1 or TSC2 but a "second hit" leads to inactivation of both alleles of one gene. Inactivation of both alleles of TSC1 or TSC2 is required for the manifestations of TS to develop [1]. The disease is transmitted in an autosomal dominant manner. Only $20 \%$ of patients with TS have a positive family history; while the other $80 \%$ of patients have de novo mutations [4]. The products of TSC1 and TSC2 are hamartin and tuberin, respectively. These two gene products inactivate Rheb, which is a protein vital in regulating cellular growth and cell cycle progression, and thereby, inhibit mTOR (mammalian target of rapamycin), a serine/threonine protein kinase. The mTOR forms either mTOR complex 1 or complex 2. The mTOR complex 1 is rapamycin-sensitive; mTOR complex 2 is rapamycin-insensitive. The mTOR complex 1 has an integral role in cell growth, proliferation, and angiogenesis. The absence of hamartin or tuberin, due to a mutation in TSC 1 or TSC2 respectively, leads to unregulated increase in mTOR activity which in turn leads to the manifestations and progression of different Tuberous sclerosis complex (TSC) lesions [1].

\section{Renal manifestations of tuberous sclerosis}

The renal manifestations of TS are either mesenchymal in origin (angiomyolipomas) or epithelial in origin (carcinomas, oncocytomas, or cysts). By far, renal angiomyolipomas have been the most studied of all renal manifestations of TS. Renal angiomyolipomas are non malignant lesions composed of vessels, smooth muscle, and fat. The incidence of renal angiomyolipomas in patients with TS is roughly $55-75 \%$ [4]. Renal angiomyolipomas are the second leading cause of death in patients with TS [4]. Initial management of renal angiomyolipomas is monitoring of growth with periodic ultrasound (US) or cross sectional imaging studies 


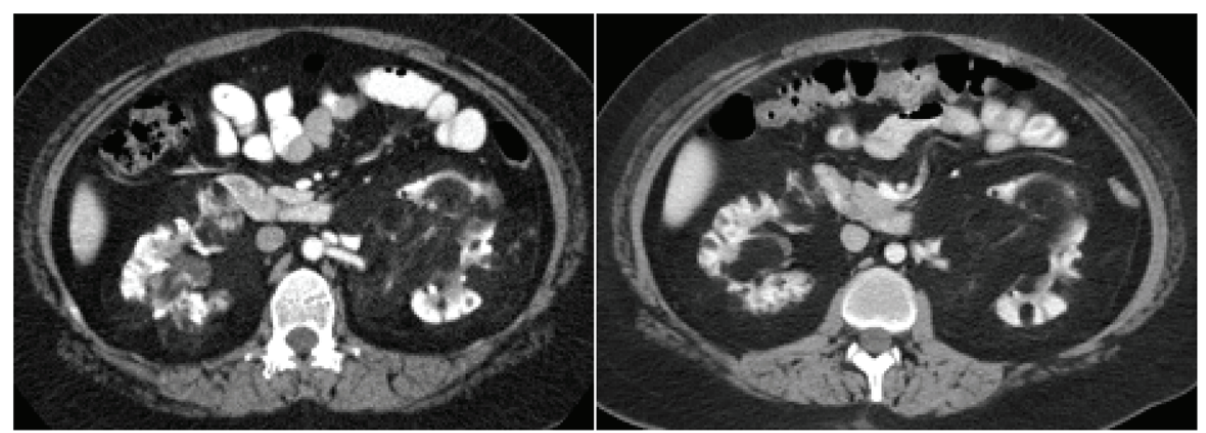

Figure 1. Right renal hilar AML lesion pre and post everolimus therapy for eleven months. The right renal hilar AML lesion measured $2.8 \mathrm{~cm} \times 2.1 \mathrm{~cm}$ in cross section and $1.8 \mathrm{~cm} \mathrm{CC}$. This is seen in the picture on the left. This lesion increased in size to $3.2 \mathrm{~cm} \times 2.2 \mathrm{~cm}$ in cross section and $2.4 \mathrm{~cm} \mathrm{CC}$ on the CT scan of the abdomen done eleven months after everolimus treatment and is seen in the picture on the right. Its density was $5 \mathrm{HU}$ in the initial CT scan of the abdomen and had decreased to $-86 \mathrm{HU}$ in the CT scan done eleven months after initiation of everolimus.

such as contrast enhanced MRI or CT scan. Major risks associated with renal angiomyelolipomas include hemorrhage, compression of adjacent renal parenchyma, compression of adjacent organs, and kidney failure. Lesions larger than 4 $\mathrm{cm}$ in size are more likely to develop hemorrhage compared to smaller ones [4]. Progressive growth of renal angiomyolipomas or conversion to malignant renal tumors may lead to loss of renal function in approximately $5 \%$ of TS patients [2]. To date embolization and/or surgical resection have been the mainstay of treatment of renal angiomyolipomas [1].

\section{Management of patients with TS with $\mathrm{mTOR}$ inhibitors}

In the last decade, mTOR inhibitors have emerged as a potential systemic treatment strategy to halt the growth of renal angiomyolipomas and to prevent life threatening complications such as bleeding, which becomes more prevalent with increase in the size of these lesions [1]. The mTOR inhibitors bind the receptor protein FKBP-12. Immunosuppressant drugs also bind FKBP-12. The binding of MTOR inhibitors to FKBP-12 forms an inhibitory complex with high affinity for mTOR complex 1 but not for mTOR complex 2 [4]. The proteins S6K1 and 4EBP1 play a key role in coordinating translation factors and ribosomal activity. The inhibitory complex formed from the binding of an mTOR inhibitor and FKBP-12 affects S6K1 and 4EBP1 which in turn results in inhibition of DNA synthesis and impairment of cell proliferation in renal angiomyiolipomas [4]. Case reports in patients with TS have shown that treatment with the mTOR inhibitor sirolimus (rapamycin) effectively controls renal
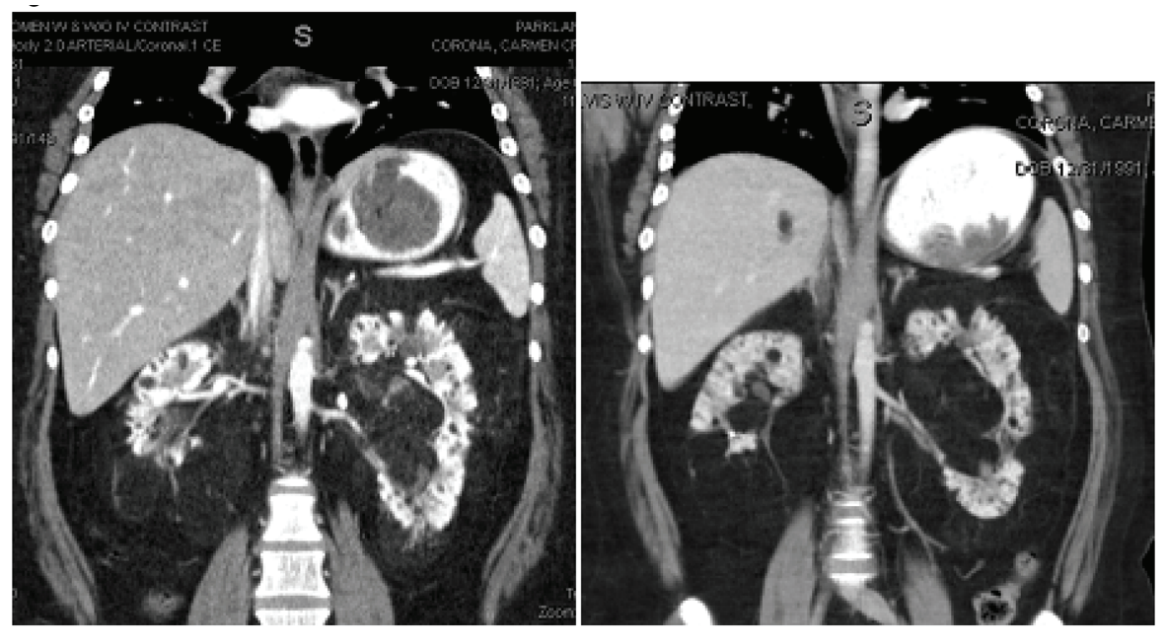

Figure 2. Left renal hilar lesions pre and post everolimus therapy for eleven months. In the left kidney, the renal hilum was markedly splayed by a conglomerate of fatty AML lesions seen in the image on the left. The image on the right depicts an increasing amount of fat content and a decrease in vascularity after eleven months of treatment with everolimus. 


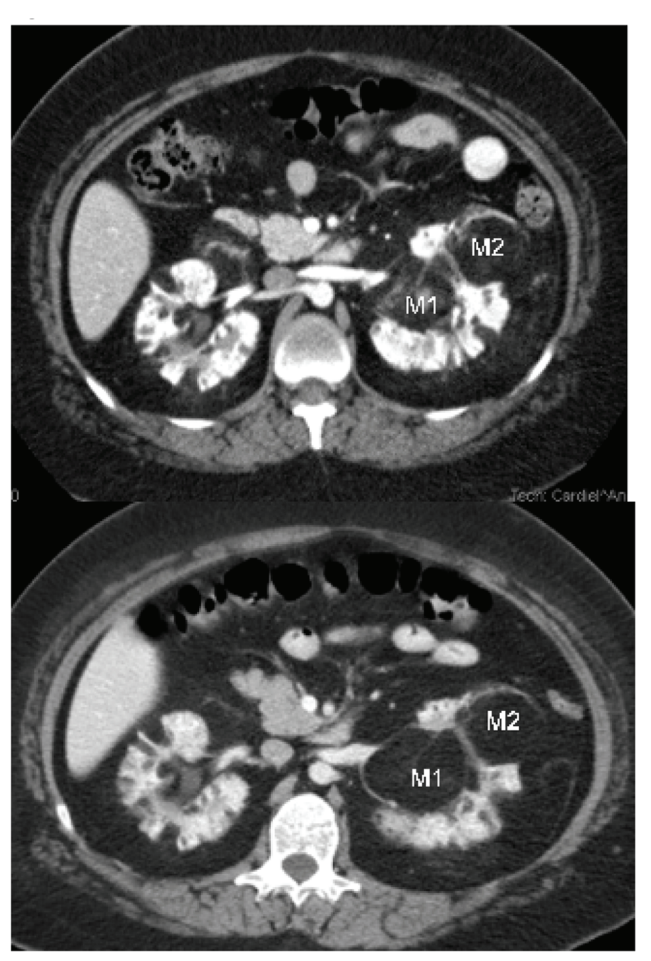

Figure 3. Left renal hilar AML lesions. M1: An AML lesion in the left renal hilum projecting medially, measured 4.9 $\mathrm{cm} \mathrm{AP}$ and $8.7 \mathrm{~cm} \times 4.9 \mathrm{~cm}$ on coronal view after eleven months of therapy with everolimus, seen in the second image. This compared to $4.8 \mathrm{~cm} \mathrm{AP}$ and $7.9 \mathrm{~cm} \times 4.8 \mathrm{~cm}$ on coronal view on the initial CT scan of the abdomen depicted in the first image. After eleven months of treatment with everolimus the density of the left renal hilar AML lesion described above decreased from $-54 \mathrm{HU}$ to $-86 \mathrm{HU}$ and the mass appeared less vascular. M2: A mass in the mid segment of the left kidney projecting anteriorly, measured $3.9 \mathrm{~cm} \mathrm{AP}$ and $4.8 \mathrm{~cm} \times 5.3 \mathrm{~cm}$ on coronal view after eleven months of therapy with everolimus depicted in the second image. This compared to $4.8 \mathrm{~cm} \mathrm{AP}$ and $4.1 \mathrm{~cm} \times 4.7 \mathrm{~cm}$ on coronal view on initial CT scan of the abdomen which is depicted in the first image. After eleven months of treatment with everolimus the density of the left renal hilar AML lesion described above decreased from $-77 \mathrm{HU}$ to $-96 \mathrm{HU}$ and the mass appeared less vascular.

angiomyolipoma-associated bleeding, halts growth, and reduces the volume of renal angiomyolipomas [4]. These studies suggest a role of mTOR inhibition in the regression of renal angiomyolipomas and in the management of extra renal manifestations of TS. Everolimus is an oral derivative of sirolimus (rapamycin) with improved pharmacokinetics including better bioavailability and CNS penetration compared to sirolimus (rapamycin) [4].

The present report describes two young adult female patients with sporadic TS, bilateral renal angiomyolipomas (AML) and other manifestations characteristic of TS treated with everolimus $10 \mathrm{mg}$ per mouth daily. The radiographic changes of renal angiomyolipoma lesions and clinical out- come with everolimus are described and discussed.

\section{Case Report}

\section{Case 1}

The first patient is a 20-year-old Hispanic female with a history of sporadic TS who initially presented to our center to establish care for the management of her TS and bilateral renal AML. The patient was diagnosed with TS after presenting with a seizure at 2 months of age. She had a prior embolization of an AML lesion of the right kidney three years prior at an outside facility. On presentation she had multiple skin angiofibromas predominantly on her face and displayed mild cognitive deficits. An MRI of the brain showed cortical tubers, white matter lesions, and subependymal hamartomas which were largely unchanged from prior scans. SEGA lesions were not identified. Contrast enhanced computed tomography (CT) scan of the chest did not show evidence of lung lymphangiomatosis or cardiac rhabdomyosarcoma. Several small AML lesions of the liver were identified. The patient initiated treatment with everolimus $10 \mathrm{mg}$ per day. She tolerated oral mTOR inhibitor therapy very well without serious adverse events. The main side effect was a mild and transient rise in the serum triglyceride level to $204 \mathrm{mg} /$ $\mathrm{dL}$ compared to $131 \mathrm{mg} / \mathrm{dL}$ one month prior. The hypertriglyceridemia improved with diet control. Six months after initiation of therapy, there was noticeable improvement in the patient's skin angiofibromas. A baseline cognitive assessment was not obtained; therefore, we do not have an objective comparison to validate the improvement in the patient's mood and cognition reported by the patient's caregiver. Thirteen months after initiation of everolimus, a contrast enhanced MRI of the brain was unchanged compared to the baseline study. New brain lesions were not identified. Contrast enhanced CT scan of the abdomen and pelvis was obtained at two weeks and eleven months post therapy initiation. In the right kidney, a fatty mass arising from the lower pole and extending into the perinephric space was seen. The mass involved the lower half of the right kidney. Due to its amorphous shape and indistinct margins, accurate measurements could not be obtained. The mass had an initial density of -88 Hounsfield units (HU), slightly higher than the density of the surrounding perinephric fat. The mass was consistent with a large renal AML lesion. After eleven months of treatment with everolimus, the density of the right renal AML lesion decreased to $-99 \mathrm{HU}$, likely reflecting an increase in fatty component. The renal mass had become isodense with the surrounding perinephric fat and its margins were no longer visible. There also appeared to be less linear densities suggestive of a reduction in vascularity. A right renal hilar AML lesion, measuring $2.8 \mathrm{~cm} \times 2.1 \mathrm{~cm}$ in cross section and 1.8 $\mathrm{cm} \mathrm{CC}$ in the initial CT scan of the abdomen increased in size 


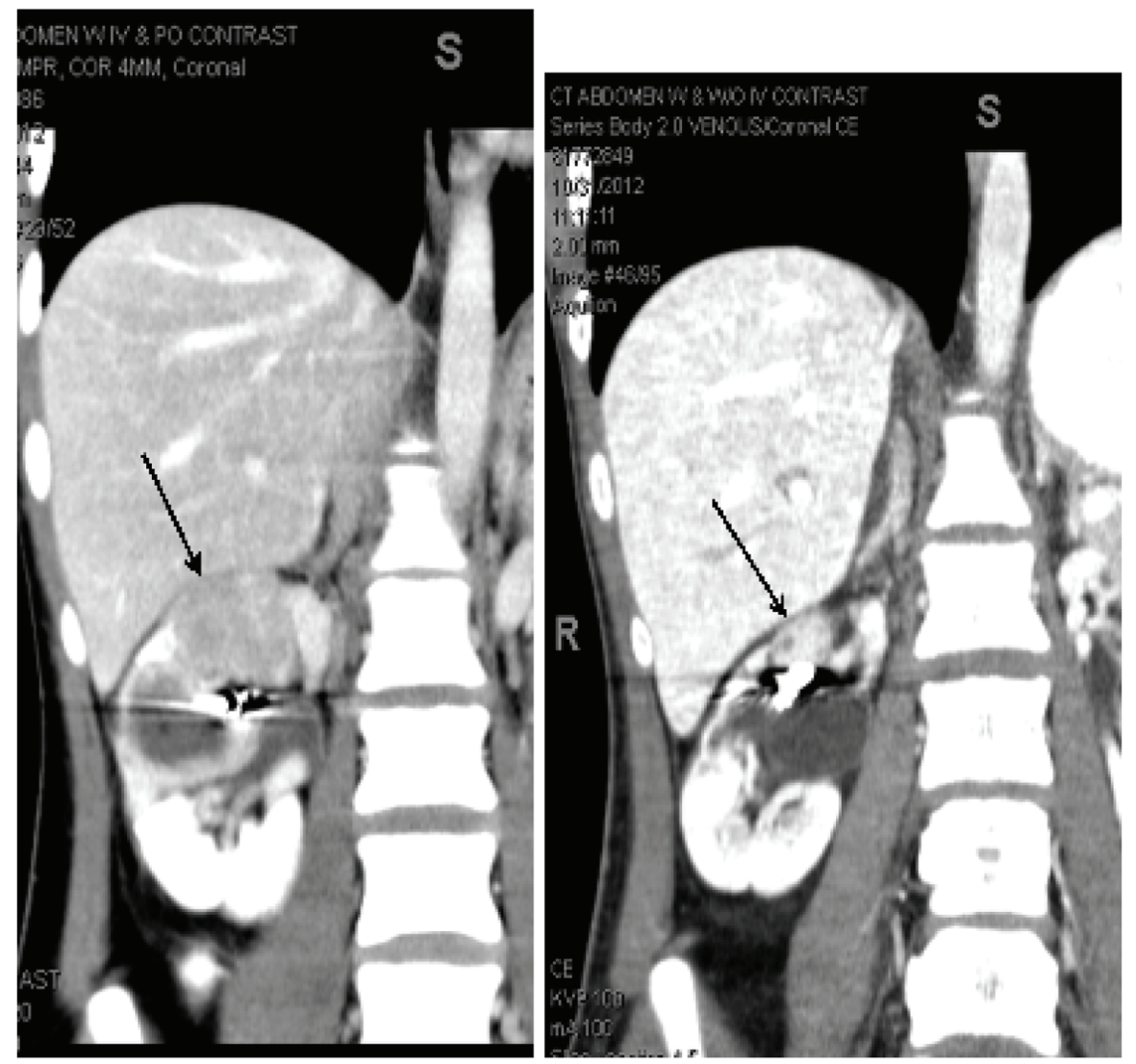

Figure 4. AML lesions of mid segment of right kidney. The image on the left showed an AML lesion involving the mid segment of the right kidney measuring $4.5 \mathrm{~cm} \times 4.5 \mathrm{~cm}$ in cross section and $3.5 \mathrm{~cm} \mathrm{CC}$. Three months after treatment with everolimus, this AML lesion had decreased in size to $1.9 \mathrm{~cm} \times 1.9 \mathrm{~cm}$ in diameter and $1.8 \mathrm{~cm} \mathrm{CC}$. Five months after treatment with everolimus, the same lesion was stable and measured $2.0 \mathrm{~cm} \times 2.0 \mathrm{~cm}$ in diameter and $1.8 \mathrm{~cm}$ CC. This is depicted in the picture on the right. Of note, the radiographic density of the right renal mid segment AML lesion increased during treatment with everolimus.

to $3.2 \mathrm{~cm} \times 2.2 \mathrm{~cm}$ in cross section and $2.4 \mathrm{~cm} \mathrm{CC}$ on the $\mathrm{CT}$ scan of the abdomen done eleven months after oral mTOR inhibitor therapy initiation. This right renal hilar AML lesion also appeared more fatty and less vascular. Its density was 5 $\mathrm{HU}$ in the initial CT scan of the abdomen and had decreased to $-86 \mathrm{HU}$ in the $\mathrm{CT}$ scan done eleven months after initiation of everolimus (Fig. 1). Numerous, smaller renal cysts appeared grossly unchanged in the initial and follow up CT scans of the abdomen. In the left kidney, the renal hilum was markedly splayed by a conglomerate of fatty AML lesions, which also showed an increasing amount of fat content and a decrease in vascularity in the CT scan of the abdomen done after eleven months of treatment with everolimus (Fig. 2). An AML lesion in the left renal hilum projecting medially, measured $4.9 \mathrm{~cm}$ AP and $8.7 \mathrm{~cm} \times 4.9 \mathrm{~cm}$ on coronal view in the eleven month post therapy CT scan of the abdomen compared to $4.8 \mathrm{~cm}$ AP and $7.9 \mathrm{~cm} \times 4.8 \mathrm{~cm}$ on coronal view on the initial CT scan of the abdomen. After eleven months of treatment with everolimus the density of the left renal hilar AML lesion described above decreased from -54 HU to -86 HU consistent with an increase in fatty content. This mass also appeared less vascular (Fig. 3, depicted as M1). A mass in the mid segment of the left kidney projecting anteriorly, measured $3.9 \mathrm{~cm}$ AP and $4.8 \mathrm{~cm} \times 5.3 \mathrm{~cm}$ on coronal view in the eleven month post therapy CT scan of the abdomen compared to $4.8 \mathrm{~cm} \mathrm{AP}$ and $4.1 \mathrm{~cm} \times 4.7 \mathrm{~cm}$ on coronal view on initial CT scan of the abdomen. After eleven months of treatment with everolimus the density of the left renal hilar AML lesion described above decreased from -77 HU to -96 HU. This mass also appeared less vascular (Fig. 3) (depict as M2). The mass in the mid lower segment, measured $5.4 \mathrm{~cm}$ $\mathrm{AP}$ and $5.7 \mathrm{~cm} \times 5.3 \mathrm{~cm}$ oblique on coronal view on CT scan completed eleven months post therapy compared to $4.8 \mathrm{~cm}$ $\mathrm{AP}$ and $5.8 \mathrm{~cm} \times 5.1 \mathrm{~cm}$ oblique on coronal view on initial CT scan of the abdomen. After eleven months of treatment with everolimus the density of the left renal hilar AML lesion described above decreased from -78HU to -94HU. It also appeared less vascular.

\section{Case 2}

The second patient is a 24-year-old Asian female who was 


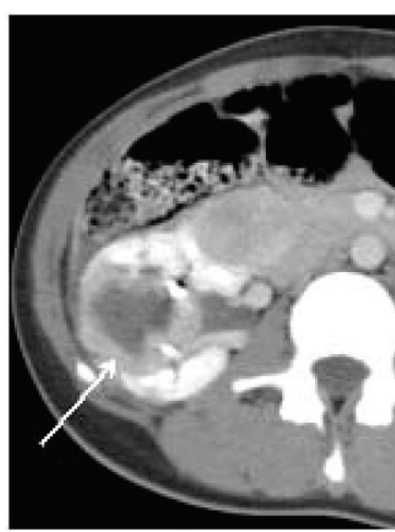

A

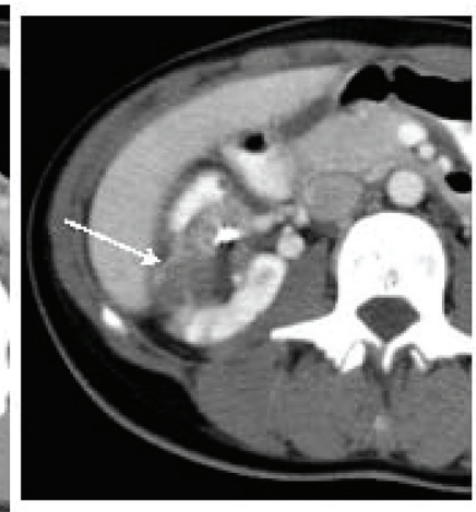

B

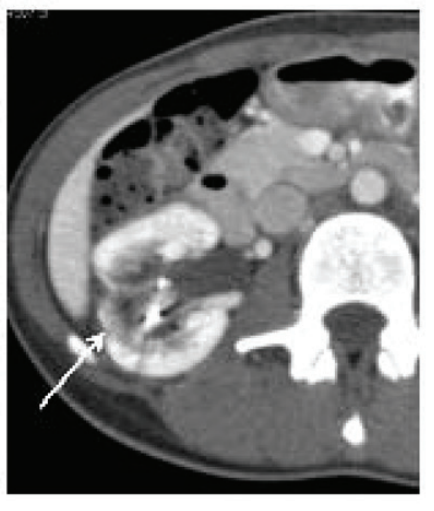

C

Figure 5. AML lesions of middle of right kidney. An AML lesion located in the middle of the right kidney measured $4.0 \mathrm{~cm} \times 4.5$ $\mathrm{cm}$ on the initial CT scan prior to everolimus therapy (image A). Three months after treatment it measured $2.3 \mathrm{~cm} \times 2.8 \mathrm{~cm}$ in cross section (image B) and was difficult to delineate at five months post therapy initiation with everolimus (image C).

diagnosed with sporadic TS at the age of 3 months after presenting with seizures. More recently she had developed severe and sudden abdominal pain due to acute bleeding into a right renal pseudoaneurysm associated with a large AML lesion for which she underwent emergent right renal coil embolization. The patient reported a history of chronic seizure disorder. A contrast enhanced MRI of the brain showed multiple cortical and subcortical tubers as well lesions highly suggestive of SEGAs. A contrast enhanced CT scan of the chest showed multiple pulmonary cysts and nodules not characteristic of LAMs. Also, the patient had innumerable facial angiofibromas. She underwent a repeat embolization of the right kidney AML lesions five months after the initial embolization. Subsequently she was prescribed oral everolimus 10 milligrams (mg) daily. Treatment was associated with mild to moderate superficial oral ulcerations one month after treatment initiation of everolimus for which the oral mTOR inhibitor was held for one week. The patient had complete resolution of oral mucosal superficial ulceration and reinitiated treatment with everolimus $10 \mathrm{mg}$ per mouth daily. Six weeks after initiation of everolimus, contrast enhanced MRI of the brain showed stable TS lesions. Contrast enhanced CT scan of the abdomen and pelvis obtained at one month and five months after initiation of everolimus were compared to a baseline CT scan of the abdomen and pelvis obtained prior to initiation of everolimus. The baseline contrast enhanced $\mathrm{CT}$ scan of the abdomen showed an AML lesion involving the mid segment of the right kidney measuring $4.5 \mathrm{~cm} \times 4.5$ $\mathrm{cm}$ in cross section and $3.5 \mathrm{~cm} \mathrm{CC}$. Three months after treatment with everolimus, this AML lesion had decreased in size to $1.9 \mathrm{~cm} \times 1.9 \mathrm{~cm}$ in diameter and $1.8 \mathrm{~cm} \mathrm{CC}$. Five months after treatment with everolimus, the same lesion was stable and measured $2.0 \mathrm{~cm} \times 2.0 \mathrm{~cm}$ in diameter and $1.8 \mathrm{~cm} \mathrm{CC}$. Of note, the radiographic density of the right renal mid segment AML lesion increased during treatment with everolimus. The radiographic density was $240 \mathrm{HU}$ after five months of treatment with everolimus, markedly increased from 125 $\mathrm{HU}$ and $107 \mathrm{HU}$ in the CT scans obtained after three months and prior to initiation of everolimus therapy respectively. The increase in the radiographic density of the right renal AML lesion suggests a decrease in its fatty component and/ or increase in vascularity (Fig. 4). An AML lesion located in the mid right kidney measuring $4.0 \mathrm{~cm} \times 4.5 \mathrm{~cm}$ on the initial CT scan prior to everolimus therapy was noted to have a central lucency, which indicated either necrosis or hemorrhage. Three months after treatment it measured $2.3 \mathrm{~cm} \times 2.8 \mathrm{~cm}$ in cross section was difficult to delineate at five months post therapy initiation with everolimus (Fig. 5).

A large solid mass involving the lower pole of the right kidney became cystic after three months of treatment with everolimus. It appears as two adjacent but separate cysts. The first of the two lesions located in the hilum of the right kidney, projecting anteriorly, had decreased in size to $1.5 \mathrm{~cm}$ $\times 1.9 \mathrm{~cm}$ in cross section and $1.8 \mathrm{~cm} \mathrm{CC}$ five months after treatment with everolimus compared to $2.1 \mathrm{~cm} \times 2.2 \mathrm{~cm}$ in cross section and $2.4 \mathrm{~cm} \mathrm{CC}$ three months after initiation of everolimus. Radiographic density of this lesion was $70 \mathrm{HU}$ and $42 \mathrm{HU}$, five and three months after treatment with everolimus respectively. A cyst located adjacent to this AML lesion, which was previously $2.0 \mathrm{~cm} \times 2.4 \mathrm{~cm}$ cross section and $2.2 \mathrm{~cm} \mathrm{CC}$, also decreased in size to $1.4 \mathrm{~cm} \times 1.8 \mathrm{~cm}$ in cross section and $1.4 \mathrm{~cm} \mathrm{CC}$ on the CT completed at five months post treatment initiation. Its density also increased from 46 HU to $63 \mathrm{HU}$ (Fig. 6). There were two adjacent AML lesions present in the mid segment of the left kidney. The larger, more posterior lesion measured $2.7 \mathrm{~cm} \times 2.5 \mathrm{~cm}$ cross section and $3.7 \mathrm{~cm}$ oblique on the coronals on the baseline CT conducted prior to everolimus initiation. The lesion had reduced in size to $2.4 \mathrm{~cm} \times 1.7 \mathrm{~cm}$ in cross section and $2.6 \mathrm{~cm}$ in long oblique (coronals) three months post therapy initiation. At five months, this lesion had further decreased in size to $2.1 \mathrm{~cm} \times 1.7 \mathrm{~cm}$ in cross section and longest oblique (on 


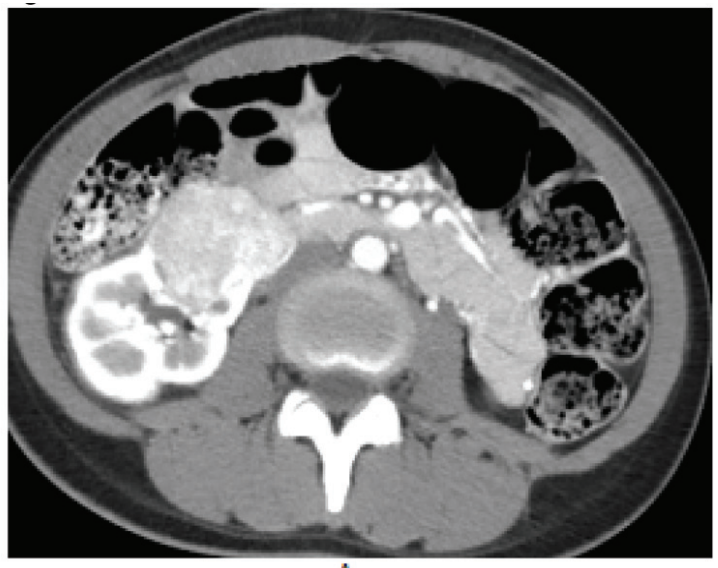

A

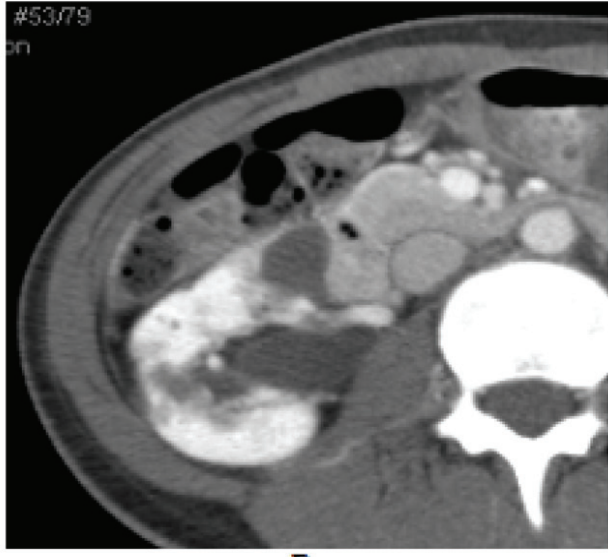

B

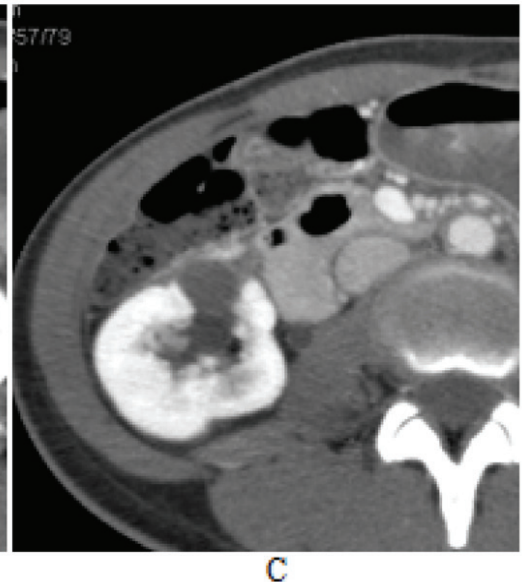

C

Figure 6. AML lesion of lower pole of right kidney. A large solid mass involving the lower pole of the right kidney (image A) becomes cystic after three months of everolimus treatment (image B). It appears as two adjacent but separate cysts. The first of the two lesions located in the hilum of the right kidney, projecting anteriorly, had decreased in size to $1.5 \mathrm{~cm} \times 1.9 \mathrm{~cm}$ in cross section and $1.8 \mathrm{~cm} \mathrm{CC}$ five months after treatment with everolimus (image C) compared to $2.1 \mathrm{~cm} \times 2.2 \mathrm{~cm}$ in cross section and $2.4 \mathrm{~cm} \mathrm{CC}$ three months after initiation of everolimus (image B). A cyst located adjacent to this AML lesion, which was previously $2.0 \mathrm{~cm} \times 2.4 \mathrm{~cm}$ cross section and $2.2 \mathrm{~cm} \mathrm{CC}$ (image B), also decreased in size to $1.4 \mathrm{~cm} \times 1.8 \mathrm{~cm}$ in cross section and $1.4 \mathrm{~cm} \mathrm{CC}$ on the $\mathrm{CT}$ completed at five months post treatment (image C).

coronal MPR) of $2.5 \mathrm{~cm}$. Its density progressively increased, from $131 \mathrm{HU}$ at baseline to $144 \mathrm{HU}$ at three months to 240 $\mathrm{HU}$ at five months post therapy initiation. A smaller spherical lesion was located anterior to this AML lesion. This small spherical lesion was $1.4 \mathrm{~cm}$ in diameter initially but also was noted to reduce in size to $0.95 \mathrm{~cm}$ then $0.77 \mathrm{~cm}$ at three and five months post everolimus initiation respectively. The density of this lesion also increased from $100 \mathrm{HU}$ initially to 148 $\mathrm{HU}$ at three months and $224 \mathrm{HU}$ at five months.

\section{Discussion}

In the past decade, progress has been made in understanding the role of the mTOR signaling pathway and its inhibition in patients with TS. Regressive effects of renal AML lesions and improvement of other TS-associated extra renal manifestations have been demonstrated in patients treated with the mTOR inhibitor sirolimus. Treatment with sirolimus has been associated with regression and control of renal AML lesions during treatment in patients with TS. In these patients sirolimus withdrawal led to subsequent regrowth of the AML lesions $[5,6]$. In a study conducted by Bissler et al, a reduction of AML volume of $47 \%$ was seen at one year of treatment with sirolimus; while a study by Davies et al showed a volume reduction of $60 \%[3,5]$. Lower drug levels were achieved in the latter study. An increase in the volume of AML lesions from $53 \%$ to $86 \%$ was seen over the course of 12 months following withdrawal of sirolimus [4].

Vascular endothelial growth factor (VEGF) plays a fundamental role in regulating angiogenesis. A multicenter prospective phase 2 clinical trials of 36 adults with TSC or 
TSC/LAM treated with daily sirolimus showed a correlation between serum VEGF levels and the size of AML lesions. It was noted that serum VEGF-D levels were elevated at baseline in patients with renal AML lesions, and when a decrease in AML size was noted a corresponding decrease in VEGF levels was also noted [7].

EXIST-2 was an international, multicenter, double blinded, placebo-controlled randomized clinical trial evaluating the safety and efficacy of everolimus in adult patient patients 18 years old or older with AML associated with TSC or sporadic LAM of the lungs. A $42 \%$ objective response rate of AML lesions to everolimus was seen. Few adverse events including stomatitis, nasopharyngitis, and acne like lesions were observed. As early as 24 weeks into treatment, 55\% of patients receiving everolimus had at least a $50 \%$ reduction in sums of volumes of lesions compared to baseline. Treatment with everolimus was associated with improvement in progression of AML lesions compared to placebo. This study showed that everolimus was associated with a decreased in the volume of renal AML lesions and decreased the rate of progression of AML lesions. Treatment with everolimus was associated with an acceptable safety profile [8]. There are a few ongoing clinical studies evaluating the role of everolimus in the treatment of TSC patient with renal AML. Currently, a clinical trial is being conducted in patients with TSC ages 18 - 65 to examine the efficacy and safety of everolimus in the treatment of AML lesions [4]. In addition, a case report published on a patient with LAMs and lung transplantation showed a reduction of renal AML lesions when the patient was treated with everolimus [9]. The role of mTOR inhibition as induction (neoadjuvant) therapy prior to nephron sparing resection of renal AML lesions is under evaluation [10].

\section{Conclusion}

The goals of treatment with everolimus in the two patients described in the current report were to delay the progression or growth of renal AML lesions, prevent bleeding from renal AML lesions and prevent or delay the development of renal cell carcinoma. The first patient has been followed for a total of 13 months and has shown considerable improvement in fat content/density of the renal AML lesions and possibly a decrease in mass vascularity; although, the size of her lesions have been stable or have slightly increased in size. There are notable improvements in the extra renal manifestations of TS including a decrease in the size and number of facial angiofibromas as well as improvement in mood and cognition reported by the patient's care giver. In addition, her contrast enhanced MRI brain has shown stable findings with no growth or change in the suspected SEGAs.

The second patient showed considerable improvement in the size of her bilateral renal AML lesions with daily everolimus. This benefit was documented after five months of mTOR inhibitor therapy. The increased radiographic den- sity seen in the renal AML lesions with daily everolimus in this patient is not well understood. This could represent intra tumoral hemorrhage. However, the lack of acute changes is the size of renal AML lesions and the lack of symptoms like acute flank pain or drop in the hemoglobin and hematocrit do not suggest acute hemorrhage as the cause of increased radiographic density of the renal AML lesions seen during treatment with daily everolimus. To the time of the preparation of the present report neither patient has developed renal cell carcinoma or new renal hemorrhage.

Both patients showed varied responses to everolimus treatment. The first patient's response showed stability in size and marked decrease in density indicating regression of her AML lesions. The decrease in density could indicate that the lesions were becoming less vascular and more fatty which could presumably decrease the risk of subsequent bleeding episodes. The second patient's radiographic changes with daily everolimus were different. Her renal AML lesions showed increases in density which could indicate increased vascularity and decreased fatty components. This could presumably increase the risk of bleeding of renal AML lesions. The lack of acute symptoms, stable hemoglobin and hematocrit, and decrease in size of her renal AML lesions do not suggest intratumoral bleeding. Although there was marked decrease in the size of renal AML lesions with daily everolimus, the increases in radiographic density seen during treatment could indicate increased intratumoral vascularity which in turn would suggest that the mTOR inhibitor everolimus was not associated with decreases in angiogenesis. Measurements of serum VEGF levels and intratumoral vascular density in prospective clinical trials evaluating the safety and efficacy of mTOR inhibitors in patients with TS with renal AML may be helpful to understand the role of mTOR inhibitors and their effects in angiogenesis in this patient population.

The molecular mechanisms underlying the varying radiographic changes of renal AML lesions with daily everolimus therapy found in our two patients are not established. Further studies are needed to understand the radiographic changes and response rates of renal AML lesions to daily everolimus therapy. Our case report suggests that treatment with daily everolimus may lead to reduction in size and/ or decreases in density or renal AML lesions. It is unclear whether these findings will prevent or delay the development of renal cell carcinoma or prevent bleeding from renal AML lesions. The main limitation of our study is a short follow up time period on both patients. At the time of preparation of this report, both patients continue treatment with daily everolimus without serious adverse events.

\section{Acknowledgement}

None. 


\section{Competing Interests}

The authors declare that they have no competing interests.

\section{References}

1. Budde K, Gaedeke J. Tuberous sclerosis complex-associated angiomyolipomas: focus on mTOR inhibition. Am J Kidney Dis. 2012;59(2):276-283.

2. Pollizzi K, Malinowska-Kolodziej I, Stumm M, Lane H, Kwiatkowski D. Equivalent benefit of mTORC1 blockade and combined PI3K-mTOR blockade in a mouse model of tuberous sclerosis. Mol Cancer. 2009;8:38.

3. Davies DM, de Vries PJ, Johnson SR, McCartney DL, Cox JA, Serra AL, Watson PC, et al. Sirolimus therapy for angiomyolipoma in tuberous sclerosis and sporadic lymphangioleiomyomatosis: a phase 2 trial. Clin Cancer Res. 2011;17(12):4071-4081.

4. Franz DN. Everolimus: an mTOR inhibitor for the treatment of tuberous sclerosis. Expert Rev Anticancer Ther. 2011;11(8):1181-1192.

5. Bissler JJ, McCormack FX, Young LR, Elwing JM, Chuck G, Leonard JM, Schmithorst VJ, et al. Sirolimus for angiomyolipoma in tuberous sclerosis complex or lymphangioleiomyomatosis. N Engl J Med.
2008;358(2):140-151.

6. Wienecke R, Fackler I, Linsenmaier U, Mayer K, Licht $\mathrm{T}$, Kretzler M. Antitumoral activity of rapamycin in renal angiomyolipoma associated with tuberous sclerosis complex. Am J Kidney Dis. 2006;48(3):e27-29.

7. Dabora SL, Franz DN, Ashwal S, Sagalowsky A, DiMario FJ, Jr., Miles D, Cutler D, et al. Multicenter phase 2 trial of sirolimus for tuberous sclerosis: kidney angiomyolipomas and other tumors regress and VEGF- D levels decrease. PLoS One. 2011;6(9):e23379.

8. Bissler JJ, Kingswood JC, Radzikowska E, Zonnenberg BA, Frost M, Belousova E, Sauter M, et al. Everolimus for angiomyolipoma associated with tuberous sclerosis complex or sporadic lymphangioleiomyomatosis (EXIST-2): a multicentre, randomised, double-blind, placebo-controlled trial. Lancet. 2013;381(9869):817-824.

9. Bujalance-Cabrera C, Vaquero-Barrios JM, Redel-Montero J, Caballero-Ballesteros L, Requejo-Jimenez A, Santos-Luna F. Reduction in size of renal angiomyolipoma after treatment with everolimus in lung transplantation due to lymphangioleiomyomatosis. Arch Bronconeumol. 2012;48(12):479-481.

10. Staehler M, Sauter M, Helck A, Linsenmaier U, Weber L, Mayer K, Fischereder M. Nephron-sparing resection of angiomyolipoma after sirolimus pretreatment in patients with tuberous sclerosis. Int Urol Nephrol. 2012;44(6):1657-1661. 